\title{
Architectural Diversity and Elastic Networks in Hydrogen-bonded Host Frameworks: From Molecular Jaws to Cylinders
}

\author{
Matthew J. Horner, K. Travis Holman, and Michael D. Ward
}

(JA0741574)

\section{Supporting Information}

Table S1. Crystallographic information for new guest-free guanidinium organomonosulfonates.

Table S2. Crystallographic information for new guanidinium organomonosulfonate inclusion compounds. 
Table S1. Crystallographic information for new guest-free guanidinium organomonosulfonates.

\begin{tabular}{|c|c|c|c|c|c|}
\hline Compound & G2FBS & G3FBS & G4FBS & G2CBS & G3CBS \\
\hline formula & $\mathrm{C}_{7} \mathrm{H}_{10} \mathrm{~N}_{3} \mathrm{O}_{3} \mathrm{SF}$ & $\mathrm{C}_{7} \mathrm{H}_{10} \mathrm{~N}_{3} \mathrm{O}_{3} \mathrm{SF}$ & $\mathrm{C}_{7} \mathrm{H}_{10} \mathrm{~N}_{3} \mathrm{O}_{3} \mathrm{SF}$ & $\mathrm{C}_{7} \mathrm{H}_{10} \mathrm{~N}_{3} \mathrm{O}_{3} \mathrm{SCl}$ & $\mathrm{C}_{7} \mathrm{H}_{10} \mathrm{~N}_{3} \mathrm{O}_{3} \mathrm{SCl}$ \\
\hline formula wt. & 235.24 & 235.24 & 235.24 & 251.69 & 251.69 \\
\hline crystal system & monoclinic & triclinic & monoclinic & triclinic & triclinic \\
\hline space group & $C 2 / c$ & $P 1$ (bar) & $P 2_{1} / c$ & $P 1$ (bar) & $P 1$ (bar) \\
\hline color & colorless & colorless & colorless & colorless & colorless \\
\hline$a(\AA)$ & $12.561(2)$ & $7.169(5)$ & $7.469(1)$ & $7.121(5)$ & $7.316(2)$ \\
\hline$b(\AA)$ & $7.469(1)$ & $7.304(5)$ & $38.233(6)$ & $7.347(5)$ & $7.324(2)$ \\
\hline$c(\AA)$ & $22.990(3)$ & $11.532(5)$ & $11.272(2)$ & $11.661(5)$ & $12.272(3)$ \\
\hline$\alpha\left(^{\circ}\right)$ & 90 & $72.377(5)$ & 90 & $80.198(5)$ & $73.749(3)$ \\
\hline$\beta\left(^{\circ}\right)$ & $94.984(2)$ & $85.075(5)$ & $93.007(3)$ & $83.673(5)$ & $72.979(3)$ \\
\hline$\gamma\left({ }^{\circ}\right)$ & 90 & $62.944(5)$ & 90 & $63.966(5)$ & $62.076(3)$ \\
\hline$V\left(\AA^{3}\right)$ & $2148.7(5)$ & $511.6(5)$ & $3214.7(9)$ & $539.7(6)$ & $547.56(19)$ \\
\hline temp. (K) & $173(2)$ & $173(2)$ & $173(2)$ & $173(2)$ & $173(2)$ \\
\hline$Z$ & 8 & 2 & 12 & 2 & 2 \\
\hline$R_{1}[I>2 \sigma(I)]$ & 0.0382 & 0.0309 & 0.0525 & 0.0312 & 0.0389 \\
\hline$w R_{2}[I>2 \sigma(I)]$ & 0.0906 & 0.0826 & 0.1375 & 0.08646 & 0.1047 \\
\hline G.O.F. & 1.071 & 1.047 & 1.155 & 1.080 & 1.097 \\
\hline Compound & G2MBS & G4IBS & G4IPBS & G4TBBS & G4NBS \\
\hline formula & $\mathrm{C}_{8} \mathrm{H}_{13} \mathrm{~N}_{3} \mathrm{O}_{3} \mathrm{~S}$ & $\mathrm{C}_{7} \mathrm{H}_{10} \mathrm{~N}_{3} \mathrm{O}_{3} \mathrm{SI}$ & $\mathrm{C}_{10} \mathrm{H}_{17} \mathrm{~N}_{3} \mathrm{O}_{3} \mathrm{~S}$ & $\mathrm{C}_{11} \mathrm{H}_{19} \mathrm{~N}_{3} \mathrm{O}_{5} \mathrm{~S}$ & $\mathrm{C}_{7} \mathrm{H}_{10} \mathrm{~N}_{4} \mathrm{O}_{5} \mathrm{~S}$ \\
\hline formula wt. & 231.27 & 343.14 & 259.33 & 279.75 & 262.25 \\
\hline crystal system & orthorhombic & orthorhombic & orthorhombic & orthorhombic & monoclinic \\
\hline space group & Pnma & Pnma & Pbca & Pbca & $P 2_{1} / C$ \\
\hline color & colorless & colorless & colorless & colorless & colorless \\
\hline $\mathrm{a}(\AA)$ & $16.315(2)$ & $22.527(5)$ & $14.501(5)$ & $12.377(2)$ & $11.2544(9)$ \\
\hline $\mathrm{b}(\AA)$ & $7.515(1)$ & $7.555(5)$ & $12.686(5)$ & $22.114(3)$ & $7.7474(6)$ \\
\hline$c(\AA)$ & $9.277(1)$ & $6.765(5)$ & $47.584(5)$ & $56.385(9)$ & $13.095(1)$ \\
\hline$\alpha\left({ }^{\circ}\right)$ & 90 & 90 & 90 & 90 & 90 \\
\hline$\beta\left(^{\circ}\right)$ & 90 & 90 & 90 & 90 & $110.850(1)$ \\
\hline$\gamma\left({ }^{\circ}\right)$ & 90 & 90 & 90 & 90 & 90 \\
\hline$V\left(\AA^{3}\right)$ & $1137.4(3)$ & $1151(1)$ & $8754(5)$ & $15432(4)$ & $1067.0(1)$ \\
\hline temp. $(\mathrm{K})$ & $173(2)$ & $173(2)$ & $173(2)$ & $173(2)$ & $173(2)$ \\
\hline Z & 4 & 4 & 8 & 8 & 4 \\
\hline $\mathrm{R} 1[\mathrm{I}>2 \sigma(\mathrm{I})]$ & 0.0402 & 0.0443 & 0.0774 & 0.1097 & 0.0329 \\
\hline wR2 $[I>2 \sigma(I)]$ & 0.0967 & 0.1068 & 0.2231 & 0.2695 & 0.0954 \\
\hline G.O.F. & 1.122 & 1.096 & 1.086 & 1.078 & 1.074 \\
\hline Compound & G2,4DMBS & G2,5DMBS & G3,4DMBS & G2,3,4TMBS & G2,4,5TMBS \\
\hline formula & $\mathrm{C}_{9} \mathrm{H}_{15} \mathrm{~N}_{3} \mathrm{O}_{3} \mathrm{~S}$ & $\mathrm{C}_{9} \mathrm{H}_{15} \mathrm{~N}_{3} \mathrm{O}_{3} \mathrm{~S}$ & $\mathrm{C}_{9} \mathrm{H}_{15} \mathrm{~N}_{3} \mathrm{O}_{3} \mathrm{~S}$ & $\mathrm{C}_{10} \mathrm{H}_{17} \mathrm{~N}_{3} \mathrm{O}_{3} \mathrm{~S}$ & $\mathrm{C}_{10} \mathrm{H}_{17} \mathrm{~N}_{3} \mathrm{O}_{3} \mathrm{~S}$ \\
\hline formula wt. & 245.30 & 245.30 & 245.30 & 259.33 & 259.33 \\
\hline crystal system & orthorhombic & orthorhombic & orthorhombic & orthorhombic & orthorhombic \\
\hline space group & $P m c 2_{1}$ & Pnma & Pnma & Pnma & Pnma \\
\hline color & colorless & colorless & colorless & colorless & colorless \\
\hline $\mathrm{a}(\AA)$ & $7.391(1)$ & $16.621(3)$ & $18.695(3)$ & $20.044(4)$ & $18.513(2)$ \\
\hline $\mathrm{b}(\AA)$ & $12.056(2)$ & $7.446(2)$ & $7.391(1)$ & $7.464(2)$ & $7.4798(8)$ \\
\hline$c(\AA)$ & $14.037(2)$ & $9.602(2)$ & $8.540(1)$ & $8.630(2)$ & $9.399(1)$ \\
\hline$\alpha\left({ }^{\circ}\right)$ & 90 & 90 & 90 & 90 & 90 \\
\hline$\beta\left(^{\circ}\right)$ & 90 & 90 & 90 & 90 & 90 \\
\hline$\gamma\left({ }^{\circ}\right)$ & 90 & 90 & 90 & 90 & 90 \\
\hline$V\left(\AA^{3}\right)$ & $1250.8(3)$ & $1188.3(4)$ & $1180.0(3)$ & $1291.1(5)$ & $1301.5(2)$ \\
\hline temp. (K) & $173(2)$ & $173(2)$ & $173(2)$ & $173(2)$ & $173(2)$ \\
\hline Z & 4 & 4 & 4 & 4 & 4 \\
\hline $\mathrm{R} 1[\mathrm{I}>2 \sigma(\mathrm{I})]$ & 0.0274 & 0.0432 & 0.0502 & 0.0474 & 0.0350 \\
\hline wR2 $[I>2 \sigma(I)]$ & 0.0688 & 0.1223 & 0.1367 & 0.1311 & 0.0946 \\
\hline G.O.F. & 1.158 & 1.043 & 1.084 & 0.960 & 1.103 \\
\hline
\end{tabular}


Table S2. Crystallographic information for new guanidinium organomonosulfonate inclusion compounds.

\begin{tabular}{|c|c|c|c|c|c|c|}
\hline Compound & G4MBS•0.5(16) & G4MBS•(22) & G4FBS•0.5(14) & G4FBS•0.5(16) & G4FBS•(20) & G4CBS·0.5(13) \\
\hline formula & $\mathrm{C}_{26} \mathrm{H}_{40} \mathrm{~N}_{6} \mathrm{O}_{3} \mathrm{~S}_{2}$ & $\mathrm{C}_{17} \mathrm{H}_{25} \mathrm{~N}_{3} \mathrm{O}_{4} \mathrm{~S}$ & $\mathrm{C}_{23} \mathrm{H}_{32} \mathrm{~N}_{6} \mathrm{O}_{6} \mathrm{~S}_{2} \mathrm{~F}_{2}$ & $\mathrm{C}_{24} \mathrm{H}_{34} \mathrm{~N}_{6} \mathrm{O}_{6} \mathrm{~S}_{2} \mathrm{~F}_{2}$ & $\mathrm{C}_{15} \mathrm{H}_{21} \mathrm{~N}_{4} \mathrm{O}_{3} \mathrm{SF}$ & $\mathrm{C}_{24} \mathrm{H}_{34} \mathrm{~N}_{6} \mathrm{O}_{6} \mathrm{~S}_{2} \mathrm{Cl}_{2}$ \\
\hline formula wt. & 596.78 & 367.46 & 590.69 & 604.71 & 356.42 & 637.61 \\
\hline crystal system & orthorhombic & orthorhombic & monoclinic & orthorhombic & orthorhombic & orthorhombic \\
\hline space group & Pna2 $_{1}$ & $P 2_{1} 2_{1} 2_{1}$ & $P 2_{1} / n$ & Pna2 $_{1}$ & $P 2_{1} 2_{1} 2_{1}$ & Pna2 $_{1}$ \\
\hline color & colorless & colorless & colorless & colorless & colorless & colorless \\
\hline$a(\AA)$ & $18.757(3)$ & $7.4622(4)$ & $7.429(1)$ & $32.57(1)$ & $7.5332(3)$ & $18.398(1)$ \\
\hline$b(\AA)$ & $23.471(4)$ & $11.8703(6)$ & $15.813(2)$ & $7.455(3)$ & $11.9412(5)$ & $22.824(1)$ \\
\hline$c(\AA)$ & $7.487(1)$ & $22.107(1)$ & $12.379(2)$ & $12.308(5)$ & $20.2060(9)$ & $7.7169(4)$ \\
\hline$\alpha\left(^{\circ}\right)$ & 90 & 90 & 90 & 90 & 90 & 90 \\
\hline$\beta\left(^{\circ}\right)$ & 90 & 90 & $90.603(3)$ & 90 & 90 & 90 \\
\hline$\gamma\left({ }^{\circ}\right)$ & 90 & 90 & 90 & 90 & 90 & 90 \\
\hline$V\left(\AA^{3}\right)$ & $3295.8(9)$ & 1958.2(2) & $1454.2(4)$ & $2988(2)$ & $1817.6(1)$ & $3240.5(3)$ \\
\hline temp. $(\mathrm{K})$ & $173(2)$ & $173(2)$ & $173(2)$ & $173(2)$ & $173(2)$ & $173(2)$ \\
\hline$Z$ & 4 & 4 & 2 & 4 & 4 & 4 \\
\hline$R_{1}[I>2 \sigma(I)]$ & 0.0618 & 0.0402 & 0.0479 & 0.1263 & 0.0299 & 0.0498 \\
\hline G.O.F. & 1.058 & 1.081 & 1.170 & 1.088 & 1.060 & 1.037 \\
\hline Compound & G4CBS•(25) & G4BBS•(1) & G4BBS·(2) & G4BBS•(13) & G4BBS•(21) & G4BBS•(22) \\
\hline formula & $\mathrm{C}_{15} \mathrm{H}_{20} \mathrm{~N}_{5} \mathrm{O}_{5} \mathrm{SCl}$ & $\mathrm{C}_{13} \mathrm{H}_{16} \mathrm{~N}_{3} \mathrm{O}_{3} \mathrm{SBr}$ & $\mathrm{C}_{14} \mathrm{H}_{18} \mathrm{~N}_{3} \mathrm{O}_{3} \mathrm{SBr}$ & $\mathrm{C}_{24} \mathrm{H}_{34} \mathrm{~N}_{6} \mathrm{O}_{6} \mathrm{~S}_{2} \mathrm{Br}_{2}$ & $\mathrm{C}_{14} \mathrm{H}_{18} \mathrm{~N}_{3} \mathrm{O}_{4} \mathrm{SBr}$ & $\mathrm{C}_{16} \mathrm{H}_{22} \mathrm{~N}_{3} \mathrm{O}_{4} \mathrm{SBr}$ \\
\hline formula wt. & 417.87 & 368.21 & 388.28 & 726.51 & 404.28 & 432.34 \\
\hline crystal system & orthorhombic & monoclinic & orthorhombic & orthorhombic & orthorhombic & orthorhombic \\
\hline space group & $P 2_{1} 2_{1} 2_{1}$ & Cc & Pca2 $_{1}$ & $P 2_{1} 2_{1} 2_{1}$ & $P 2_{1} 2_{1} 2_{1}$ & $P 2_{1} 2_{1} 2_{1}$ \\
\hline color & colorless & colorless & colorless & colorless & colorless & colorless \\
\hline $\mathrm{a}(\AA)$ & $7.469(2)$ & $7.4786(6)$ & $19.786(3)$ & $18.520(3)$ & $7.7431(4)$ & $11.781(2)$ \\
\hline $\mathrm{b}(\AA)$ & $12.316(3)$ & $19.359(2)$ & $7.526(1)$ & $22.903(4)$ & $11.2471(6)$ & $22.579(3)$ \\
\hline$c(\AA)$ & $20.948(4)$ & $11.5539(9)$ & $11.448(2)$ & $7.771(1)$ & $19.808(1)$ & $7.502(1)$ \\
\hline$\alpha\left(^{\circ}\right)$ & 90 & 90 & 90 & 90 & 90 & 90 \\
\hline$\beta\left({ }^{\circ}\right)$ & 90 & $91.208(2)$ & 90 & 90 & 90 & 90 \\
\hline$\gamma\left({ }^{\circ}\right)$ & 90 & 90 & 90 & 90 & 90 & 90 \\
\hline$V\left(\AA^{3}\right)$ & $1926.9(7)$ & $1672.4(2)$ & $1704.7(5)$ & $3296.0(9)$ & $1725.0(2)$ & $1995.6(5)$ \\
\hline $\mathrm{R} 1[\mathrm{I}>2 \sigma(\mathrm{I})]$ & 0.0402 & 0.0444 & 0.0518 & 0.0392 & 0.0274 & 0.0566 \\
\hline$w R 2[I>2 \sigma(I)]$ & 0.0858 & 0.1154 & 0.1207 & 0.0893 & 0.0684 & 0.1425 \\
\hline G.O.F. & 0.918 & 1.000 & 0.922 & 1.042 & 1.024 & 1.043 \\
\hline Compound & G4BBS·(26) & G4IBS·(22) & G4IBS•(26) & G4EBS•(3) & G4IPBS•0.67(5) & G4IPBS•0.67(8) \\
\hline formula & $\mathrm{C}_{16} \mathrm{H}_{20} \mathrm{~N}_{5} \mathrm{O}_{3} \mathrm{SBr}$ & $\mathrm{C}_{16} \mathrm{H}_{22} \mathrm{~N}_{3} \mathrm{O}_{4} \mathrm{SI}$ & $\mathrm{C}_{16} \mathrm{H}_{20} \mathrm{~N}_{5} \mathrm{O}_{3} \mathrm{SI}$ & $\mathrm{C}_{17} \mathrm{H}_{25} \mathrm{~N}_{3} \mathrm{O}_{3} \mathrm{~S}$ & $\mathrm{C}_{92} \mathrm{H}_{141} \mathrm{~N}_{18} \mathrm{O}_{18} \mathrm{~S}_{6}$ & $\mathrm{C}_{48} \mathrm{H}_{75} \mathrm{~N}_{9} \mathrm{O}_{9} \mathrm{~S}_{3}$ \\
\hline formula wt. & 442.34 & 479.34 & 489.33 & 351.47 & 1979.65 & 1018.38 \\
\hline crystal system & orthorhombic & orthorhombic & orthorhombic & monoclinic & monoclinic & monoclinic \\
\hline space group & Pna2 $_{1}$ & $P 2_{1} 2_{1} 2_{1}$ & Pna2 $_{1}$ & Cc & $P 2_{1} / c$ & $P 2_{1} / c$ \\
\hline color & colorless & colorless & colorless & colorless & colorless & colorless \\
\hline $\mathrm{a}(\AA)$ & $21.070(2)$ & $7.5106(4)$ & $21.335(1)$ & $7.508(1)$ & $18.965(3)$ & $19.115(2)$ \\
\hline$b(\AA)$ & $12.655(1)$ & $11.8486(7)$ & $12.5958(7)$ & $20.473(4)$ & $21.733(3)$ & $21.745(2)$ \\
\hline$c(\AA)$ & $7.4141(7)$ & $22.987(1)$ & $7.4420(4)$ & $12.377(2)$ & $13.832(2)$ & $14.123(1)$ \\
\hline$\alpha\left(^{\circ}\right)$ & 90 & 90 & 90 & 90 & 90 & 90 \\
\hline$\beta\left(^{\circ}\right)$ & 90 & 90 & 90 & $92.765(3)$ & $101.781(3)$ & $101.987(1)$ \\
\hline$\gamma\left({ }^{\circ}\right)$ & 90 & 90 & 90 & 90 & 90 & 90 \\
\hline$V\left(\AA^{3}\right)$ & $1976.9(3)$ & $2045.6(2)$ & 1999.9(2) & $1900.3(6)$ & $5581(1)$ & $5742.6(8)$ \\
\hline temp. (K) & $173(2)$ & $173(2)$ & $173(2)$ & $173(2)$ & $173(2)$ & $173(2)$ \\
\hline Z & 4 & 4 & 4 & 4 & 2 & 4 \\
\hline $\mathrm{R} 1[\mathrm{I}>2 \sigma(\mathrm{I})]$ & 0.0291 & 0.0423 & 0.0196 & 0.0362 & 0.0652 & 0.0606 \\
\hline$w R 2[I>2 \sigma(I)]$ & 0.0742 & 0.1212 & 0.0497 & 0.0867 & 0.1613 & 0.1966 \\
\hline G.O.F. & 1.037 & 1.087 & 1.030 & 0.978 & 1.031 & 1.060 \\
\hline
\end{tabular}


Table S2. (continued)

\begin{tabular}{|c|c|c|c|c|c|c|}
\hline Compound & G4TBBS•0.67(1) & G4TBBS $\cdot 0.25(27)$ & G4AS·(3) & G4NBS·0.5(5) & G2MBS·0.5(9) & G3MBS•(5) \\
\hline formula & $\mathrm{C}_{45} \mathrm{H}_{69} \mathrm{~N}_{9} \mathrm{O}_{9} \mathrm{~S}_{3}$ & $\mathrm{C}_{58} \mathrm{H}_{98} \mathrm{~N}_{12} \mathrm{O}_{12} \mathrm{~S}_{4}$ & $\mathrm{C}_{16} \mathrm{H}_{23} \mathrm{~N}_{3} \mathrm{O}_{4} \mathrm{~S}$ & $\mathrm{C}_{22} \mathrm{H}_{30} \mathrm{~N}_{8} \mathrm{O}_{10} \mathrm{~S}_{2}$ & $\mathrm{C}_{26} \mathrm{H}_{40} \mathrm{~N}_{6} \mathrm{O}_{6} \mathrm{~S}_{2}$ & $\mathrm{C}_{16} \mathrm{H}_{23} \mathrm{~N}_{3} \mathrm{O}_{3} \mathrm{~S}$ \\
\hline formula wt. & 976.30 & 1283.76 & 353.44 & 630.68 & 596.78 & 337.44 \\
\hline crystal system & monoclinic & monoclinic & monoclinic & monoclinic & monoclinic & orthorhombic \\
\hline space group & $P 2_{1} / c$ & $P 2_{1} / c$ & $C c$ & $P 2_{1} / c$ & $P 2_{1} / n$ & $P 2_{1} 2_{1} 2_{1}$ \\
\hline color & colorless & colorless & colorless & colorless & colorless & colorless \\
\hline$a(\AA)$ & $19.169(2)$ & $13.668(3)$ & $7.441(1)$ & 22.982(2) & $7.2620(8)$ & $7.4439(5)$ \\
\hline $\mathrm{b}(\AA)$ & $22.004(3)$ & $12.334(2)$ & $20.364(4)$ & $7.4270(6)$ & $18.062(2)$ & $11.6454(7)$ \\
\hline$c(\AA)$ & $13.228(2)$ & $22.015(4)$ & $12.248(2)$ & $19.007(1)$ & $12.144(1)$ & $21.070(1)$ \\
\hline$\alpha\left(^{\circ}\right)$ & 90 & 90 & 90 & 90 & 90 & 90 \\
\hline$\beta\left(^{\circ}\right)$ & 103.582(2) & $91.030(3)$ & $93.075(3)$ & $114.426(1)$ & $90.069(2)$ & 90 \\
\hline$\gamma\left({ }^{\circ}\right)$ & 90 & 90 & 90 & 90 & 90 & 90 \\
\hline $\mathrm{V}\left(\AA^{3}\right)$ & $5423(1)$ & $3711(1)$ & $1853.3(6)$ & $2953.8(4)$ & $1592.8(3)$ & $1826.5(2)$ \\
\hline temp. (K) & $173(2)$ & $173(2)$ & $173(2)$ & $173(2)$ & $173(2)$ & $173(2)$ \\
\hline Z & 4 & 2 & 4 & 4 & 2 & 4 \\
\hline $\mathrm{R} 1[\mathrm{l}>2 \sigma(\mathrm{I})]$ & 0.0698 & 0.1260 & 0.0302 & 0.0726 & 0.0409 & 0.0670 \\
\hline wR2 $[I>2 \sigma(I)]$ & 0.2033 & 0.3498 & 0.0717 & 0.2623 & 0.1060 & 0.2072 \\
\hline G.O.F. & 0.975 & 1.036 & 1.035 & 1.083 & 1.105 & 0.946 \\
\hline
\end{tabular}

